\title{
Segmentação de Imagens Infravermelhas Para Detecção do Câncer de Mama Utilizando U-NET CNN
}

\author{
Matheus de Freitas Oliveira Baffa ${ }^{1}$, Alessandra Martins Coelho ${ }^{2}$, Aura Conci ${ }^{3}$ \\ ${ }^{1}$ Departamento de Computação e Matemática \\ Universidade de São Paulo (USP) \\ Ribeirão Preto, SP - Brasil \\ ${ }^{2}$ Departamento Acadêmico da Ciência da Computação \\ Instituto Federal de Educação, Ciência e Tecnologia do Sudeste de \\ Minas Gerais (IF SudesteMG) \\ Rio Pomba, MG - Brasil \\ ${ }^{3}$ Instituto de Computação \\ Universidade Federal Fluminense (UFF) \\ Niterói, RJ - Brasil \\ mbaffa@usp.br, alessandra.coelho@ifsudestemg.edu.br, aconci@ic.uff.br
}

\begin{abstract}
Breast cancer is the leading type of cancer among women. According to the World Cancer Research Fund, in 2018, over 2 million new cases were detected around the world. Despite its high occurrence, early detection provides a better prognosis and helps increases the patient's survival. Significant advances in screening techniques, such as infrared imaging, have provided a cheap and less invasive way to detect the disease. Besides, computational tools can be used to assist doctors to provide a better diagnosis. Thus, this paper presents a segmentation method based on U-Net Convolutional Neural Networks. In contrast to state-of-art, machine learning approaches have shown to be efficient for the region of interest segmentation, reaching an accuracy of $98.24 \%$ and an Intersection-Over-Union of 94.38\%. The use of this segmentation method may be very useful for classification tasks, once the region of interest is well delimited for feature extraction.
\end{abstract}

Resumo. O câncer de mama é o principal tipo de câncer entre as mulheres. De acordo com o World Cancer Research Fund, em 2018, mais de 2 milhões de novos casos foram detectados em todo o mundo. Apesar de sua alta ocorrência, a detecção precoce proporciona um melhor prognóstico e auxilia no aumento da sobrevida do paciente oncológico. Avanços significativos nas técnicas de rastreamento, como as imagens infravermelhas, forneceram uma maneira barata e menos invasiva forma de detectar a doença. Além disso, ferramentas computacionais podem ser utilizadas para auxiliar os médicos a fornecerem um melhor diagnóstico. Assim, este artigo apresenta um método de segmentação baseado em Redes Neurais Convolucionais U-Net. Em contraste com o estado da arte, as abordagens de aprendizado de máquina têm se mostrado eficientes para a segmentação da região de interesse deste trabalho, atingindo uma acurácia de 98,24\% e uma Intersecção-Sobre-União de 94,38\%. O uso deste método de segmentação pode ser muito útil para tarefas de classificação, uma vez que a região de interesse é bem delimitada para extração de características. 


\section{Introdução}

A Visão Computacional é uma área de pesquisa que relaciona técnicas de processamento e análise de imagens ao reconhecimento de padrões a partir da Inteligência Artificial. O objetivo da literatura nesse campo de pesquisa é interpretar e extrair novos conhecimentos sobre os elementos que compõem uma cena ou uma imagem digital [Conci et al. 2008].

Alinhado ao desenvolvimento de novas tecnologias, a Visão Computacional tem sido responsável pelo avanço de diferentes linhas de pesquisa. A área médica, por exemplo, tem utilizado técnicas de Visão Computacional para reconhecer padrões que permitem a identificação de pacientes infectados com COVID-19 [Ismael and Şengür 2021], na identificação de tumores cerebrais [Rehman et al. 2021] e na detecção do câncer de mama [Baffa and Lattari 2018].

No âmbito do diagnóstico do câncer de mama, as imagens médicas possuem um papel fundamental na detecção e no acompanhamento do tratamento do paciente. Os tipos de imagens mais utilizados na detecção do câncer de mama são a mamografia e a Ressonância Magnética [U.S. National Cancer Institute. 2021].

Caracterizado pelo crescimento acelerado e desordenado de células nas estruturas da mama, o câncer de mama é o tipo mais frequente entre as mulheres. O Instituto Nacional do Câncer (INCA), estimou para 2020 um total de 66.280 novos casos da doença no Brasil, com uma taxa de letalidade de aproximadamente $27 \%$ [Instituto Nacional do Cancer. 2021].

Para um melhor prognóstico da doença, é indispensável realizar a detecção precoce. Uma estimativa do Instituto Nacional de Saúde da Mulher, da Criança e do Adolescente (IFF/Fiocruz) mostrou que quando descoberto nos estágios iniciais, as chances de cura do câncer de mama podem chegar a 95\% [IFF/Fiocruz. 2021].

A termografia, exame o qual é obtido uma imagem infravermelha representando a temperatura da superfície da pele, é um tipo de imagem emergente no diagnóstico do câncer de mama [Zuluaga-Gomez et al. 2019]. Além de requerer equipamentos mais baratos para sua obtenção, as imagens infravermelhas podem detectar tumores milimétricos, devido a natureza bioquímica dos tumores [Amalu et al. 2006].

A detecção do câncer de mama pela termografia é guiada pela análise da distribuição térmica por entre as mamas. É característico do corpo humano que, ao longo da superfície do corpo, a temperatura da pele seja similar por longos períodos de observação, sendo simétricos sob o eixo sagital [Gore and Xu 2003].

O desenvolvimento de sistemas de Visão Computacional pode auxiliar no processo de identificação e diagnóstico de exames. Estes sistemas, de forma inteligente detectam padrões que permitem destingir exames provenientes de pacientes saudáveis e pacientes com tumor em alguma das estruturas que compõem as mamas. Os sistemas que auxiliam no diagnóstico médico são denominados Sistemas CADx (Computer-Aided Diagnosis).

Os Sistemas CADx, assim como os sistemas de Visão Computacional, são geralmente compostos por três etapas, sendo elas (i) a segmentação da região de interesse (Region of Interest - ROI), (ii) a construção de descritores e (iii) a detecção de padrões. A segmentação da ROI é uma etapa fundamental no processo de construção do sistema CADx, uma vez que permite o programa realizar uma análise da imagem apenas da região 
onde provavelmente vá estar o tumor. Já a construção dos vetores descritores é realizada a partir da extração de características que permitem a identificação dos elementos que compõem a imagem. E por fim, a detecção de padrões é realizada utilizando técnicas de aprendizado de máquina, que aprende regras e cria modelos de classificação a partir do descritor criado na etapa anterior.

Desta forma, neste trabalho é apresentado um método de segmentação da ROI, compreendido no âmbito do diagnóstico do câncer de mama como a região externa das mamas, desde as pregas inframamárias ao pescoço. O método proposto neste trabalho implementa uma variação da U-Net, uma Rede Neural Convolucional comumente utilizada na segmentação de lâminas de tecidos histológicos. A principal contribuição deste trabalho é o modelo de segmentação baseado em Redes Neurais Profundas.

Nas seções seguintes deste trabalho, serão abordadas as atuais soluções para o problema de segmentação de imagens infravermelhas da mama disponíveis na literatura (Seção II), bem como a descrição da base de dados (Seção III), a metodologia de segmentação utilizando as Redes Neurais Convolucionais (Seção IV), os experimentos e resultados obtidos pelo método proposto (Seção V) e, por fim, a conclusão (Seção VI).

\section{Segmentação de Termografias na Literatura}

A correta segmentação da ROI em termografias favorece uma extração de características mais eficiente, resultando em sistemas CADx com modelos de classificação mais precisos e eficazes. Tal problema de segmentação é abordado na literatura e tratado a partir do uso de técnicas convencionais de processamento de imagens. Nesta seção iremos abordar alguns destes trabalhos.

[Motta 2010] propôs em seu trabalho um método de segmentação da ROI utilizando uma sequência de técnicas de processamento de imagens. Dentre estas, o autor realiza a remoção do fundo utilizando limiarização de $O t s u$, bem como encontra o limite superior das axilas, remove os braços presentes na imagem e separa as mamas esquerda e direita utilizando técnicas como a morfologia matemática, a detecção de contornos de Canny, a transformada de Hough e limiarização com refinamento adaptativo. O autor obteve uma acurácia média de $96 \%$, uma sensibilidade de $88 \%$ e uma especificidade de 99\%, avaliando sob a base de dados DMR-UFF [Silva et al. 2014].

Posteriormente, [Marques 2012] descreve um método de segmentação, também utilizando técnicas de processamento de imagens, baseado na identificação dos limites superiores, inferiores e laterais. As principais ferramentas utilizadas pelo autor é a detecção de contornos, a limiarização, o crescimento de regiões e o ajuste de curvas por mínimo quadrado e B-Splines. O autor reporta uma acurácia média de $97 \%$, uma sensibilidade de $97 \%$ e uma especifidade de $97 \%$, por entre os experimentos realizados na base de dados DMR-UFF.

Em 2016, [Baffa et al. 2016] desenvolveram um método de segmentação baseado na técnica de limiarização por refinamento adaptativo. Neste trabalho os autores detectam as regiões externas do corpo da paciente, bem como a região inferior das mamas e, a partir do cálculo da área das pregas inframamárias, um valor de limiar é encontrado. A segmentação foi avaliada sob 283 imagens infravermelhas provenientes da base de dados DMR-UFF. Os autores reportaram uma acurácia média de $96 \%$, entre os experimentos realizados, uma sensibilidade de $98 \%$ e uma especificidade de $95 \%$. 
De maneira similar, [Marques et al. 2016] propôs o desenvolvimento de um método de segmentação baseado na aplicação de uma sequência de ferramentas de processamento de imagens. Nesta sequência, são utilizados técnicas como limiarização, clusterização, detecção de bordas e refinamento. O método proposto pelos autores foi avaliado sobre 328 imagens infravermelhas provenientes da base de dados DMR-UFF. Os autores reportaram uma acurácia de $96 \%$ e uma sensibilidade de $97 \%$.

Diferente das propostas supracitadas, [Melo et al. 2017] utilizou uma Rede Neural Convolucional para realizar a segmentação da ROI em termografias. Neste trabalho, o autor desenvolve uma Rede Neural Convolucional baseada na subarquitetura Visual Geometric Group (VGG-CNN). Para o treinamento e avaliação da Rede Neural, foram utilizadas 285 imagens provenientes da base de dados DMR-UFF.O método proposto pelos autores obteve uma taxa de acurácia média entre os experimentos de 95,61\%, uma sensibilidade de 97,44\% e uma especificidade de 94,01\%.

\section{Base de Imagens Infravermelhas da Mama}

Para realizar o treinamento e avaliação do método proposto neste trabalho, foi utilizado uma base de imagens infravermelhas pública, desenvolvida pelo Grupo de Pesquisa Visual Lab, da Universidade Federal Fluminense. A base de dados Database for Mastology Research (DMR-UFF) [Silva et al. 2014] é composta por 283 imagens infravermelhas, provenientes de pacientes saudáveis e pacientes diagnosticados com câncer de mama. Além destas, uma segmentação manual também é disponibilizada no portal do grupo de pesquisa para fins de avaliação dos métodos de segmentação automática. A Figura 1 contém algumas das imagens encontradas nesta base de dados.

Figura 1. Termografias que compõem a base de dados DMR-UFF.

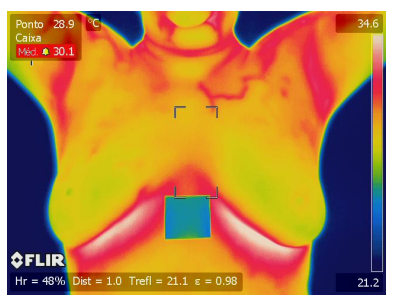

(a)

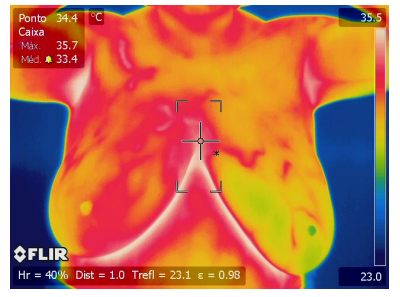

(d)

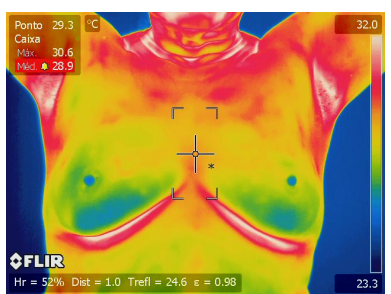

(b)

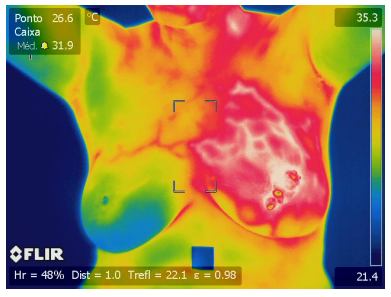

(e)

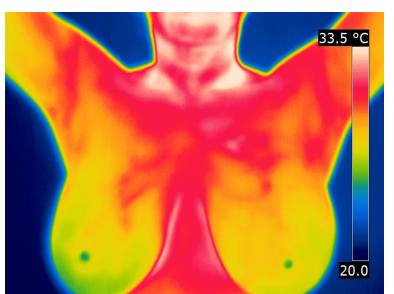

(c)

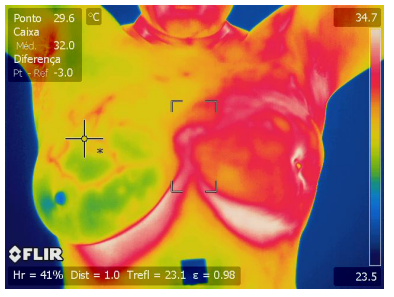

(f)

Fonte: [Silva et al. 2014].

Em (a), (b) e (c), alguns exemplos de termografias de pacientes saudáveis enquanto em (d), (e) e (f), exemplos de termografias de pacientes doentes. 


\section{Metodologia de Segmentação por U-Net}

A U-Net é uma subarquitetura de Redes Neurais Convolucionais (Convolutional Neural Network - CNN) desenvolvida com o foco na solução de problemas de segmentação de imagens biomédicas, em especial imagens contendo tecidos histológico. Caracterizada pelo formato similar a curva de uma parábola, a U-Net combina camadas de convoluções seguidas por operações de MaxPooling e Upsampling. Estas operações são responsáveis por reduzir o espaço amostral da imagem, caracterizando nas representações da arquitetura da rede como a 'descida' de uma parábola (MaxPooling), e em sequência, fazendo o caminho inverso, é realizado uma ampliação do espaço amostral, utilizando técnicas de vizinhos mais próximos (Upsampling) [Ronneberger et al. 2015].

Devido a sua alta eficiência, robustez em construir máscaras de segmentação e baixo tempo de treinamento e inferência, neste trabalho é proposto uma transposição das aplicações biomédicas da U-Net, para a construção de um algoritmo segmentador automático, aplicado à imagens médicas.

Para fins de replicabilidade, a arquitetura da U-Net proposta neste trabalho possui a seguinte configuração. Uma camada de entrada, o qual recebe uma imagem infravermelha da mama de 256x256 pixels, em escala de tons de cinza, e sua respectiva segmentação manual binária, ou groundtruth. São utilizadas 12 camadas de convolução, sendo seis para redução do espaço amostral utilizando a operação de MaxPooling com tamanho 2x2 e mais seis camadas de Upsampling, também de tamanho 2x2. As camadas de convolução utilizam a função de ativação Rectifier Linear Unit (ReLU) e extraem respectivamente 256, 128, 64, 32 e 16 filtros (MaxPooling) e 16, 32, 64, 128, 256 filtros (Upsampling), todos de tamanho $3 \times 3$. E por fim, a camada de saída é composta por uma camada de convolução 1x1 cuja função de ativação utilizada é a sigmoid.

O conjunto de hiperparametros utilizado para o treinamento foi definido empiricamente, com objetivo de maximizar a métrica Intersection-Over-Union (IOU). A Figura 2 contém um esquema gráfico representando os hiperparâmetros utilizados, bem como permite a visualização da estrutura da U-Net.

Figura 2. Customização da U-Net CNN proposta neste trabalho.
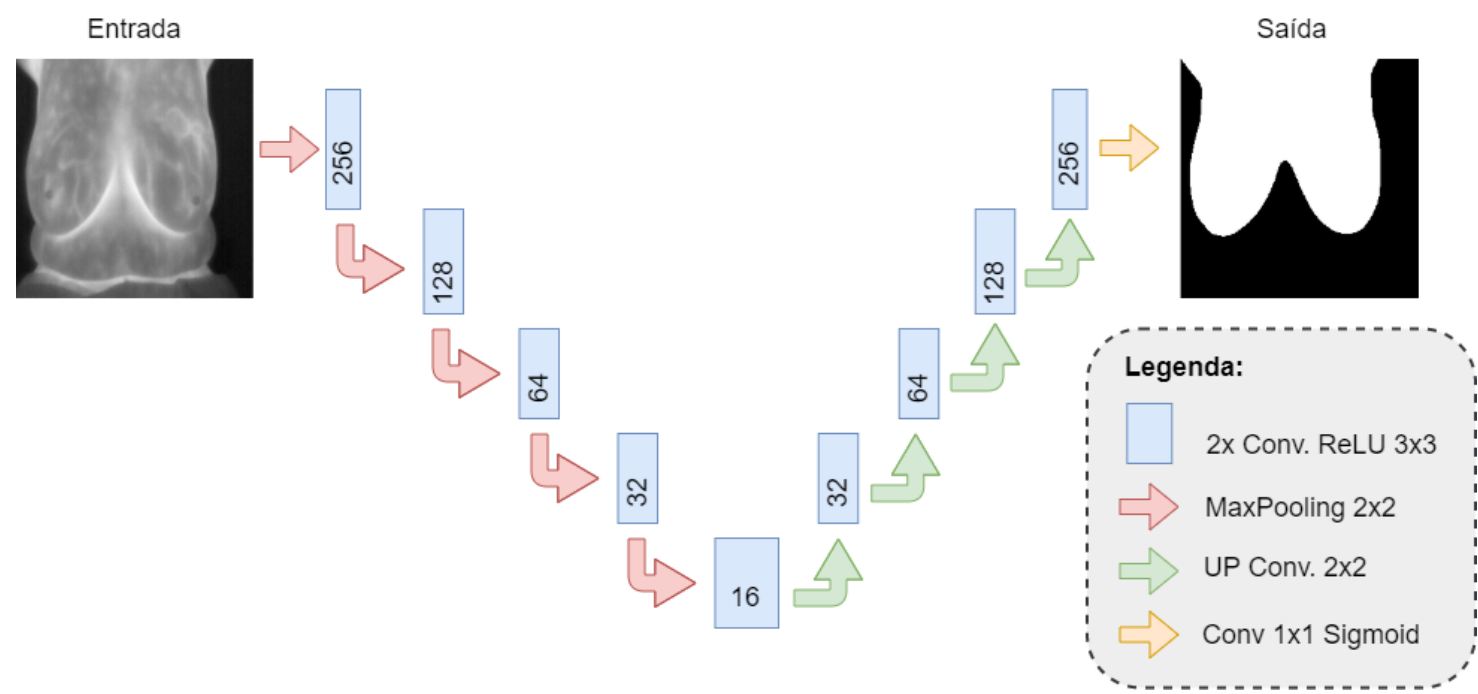

Fonte: autor 


\section{Experimentos e Resultados}

Para o desenvolvimento do método, foi utilizado um computador com um processador AMD Ryzen 7 1700, 16 GB de memória RAM, uma placa de vídeo NVIDIA GTX 1060 6GB e sistema operacional Windows 10 Pro. A implementação da U-Net foi realizada utilizando a plataforma Anaconda 3, com Python 3.7, Tensorflow 2.1 e Keras 2.3.

Os experimentos para validação do treinamento foram realizados seguindo o protocolo de experimentação Holdout Cross-Validation. Neste protocolo, parte da base de dados é utilizada para o treinamento, enquanto uma outra porção é exclusivamente utilizada para validação de forma que, as imagens utilizadas no treinamento sejam diferentes das imagens da validação. Assim, 70\% da base de dados foi utilizada para o treinamento da Rede Neural e $30 \%$ foi utilizada para validação.

Para avaliar quantitativamente o método, foram utilizadas quatro métricas, sendo elas a acurácia, a área de interseção-sobre-união (Intersection-Over-Union - IOU), a precisão e a revocação. Compreendida como uma métrica fundamental para avaliação de metodologias de segmentação, a IOU avalia a sobreposição entre máscaras de segmentação, informando a eficácia da segmentação semântica das imagens. Nesse trabalho, o processo de treinamento e experimentação buscou maximizar os valores de IOU obtidos. A IOU pode ser obtida atrás do cálculo Equação 1, a seguir:

$$
I O U=\frac{\text { Sobreposiçãa } O}{U n i \tilde{a} O}
$$

De modo geral, seguindo a arquitetura de U-Net proposta, o método convergiu ao ótimo por volta da época 30, mantendo-se estável por todo o experimento. O método proposto neste trabalho obteve uma taxa de acurácia média de $98,38 \%$ e uma baixa taxa de erro. A Figura 3 contém um gráfico com a validação do modelo de segmentação criado pela U-Net ao longo das épocas.

Figura 3. Resultados obtidos em termos de acurácia e taxa de perda por época.

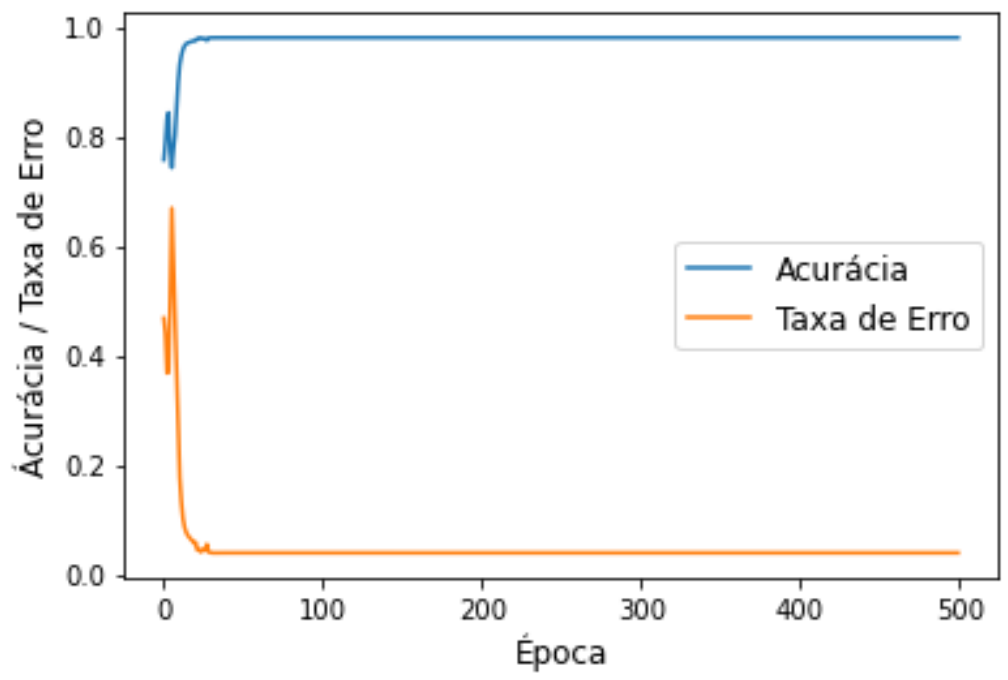

Fonte: autor.

Além da acurácia, o método proposto também obteve resultados excelentes em 
função da métrica maximizada IOU, bem como a precisão e a revocação, atingindo uma IOU média de $94,38 \%$, uma precisão média de $98,86 \%$ e uma revocação de $98,46 \%$. A Figura 4 contém um gráfico ilustrando a validação do modelo de segmentação em termos das métricas apresentadas.

Figura 4. Resultados obtidos em termos de precisão, revocação e IOU por época.

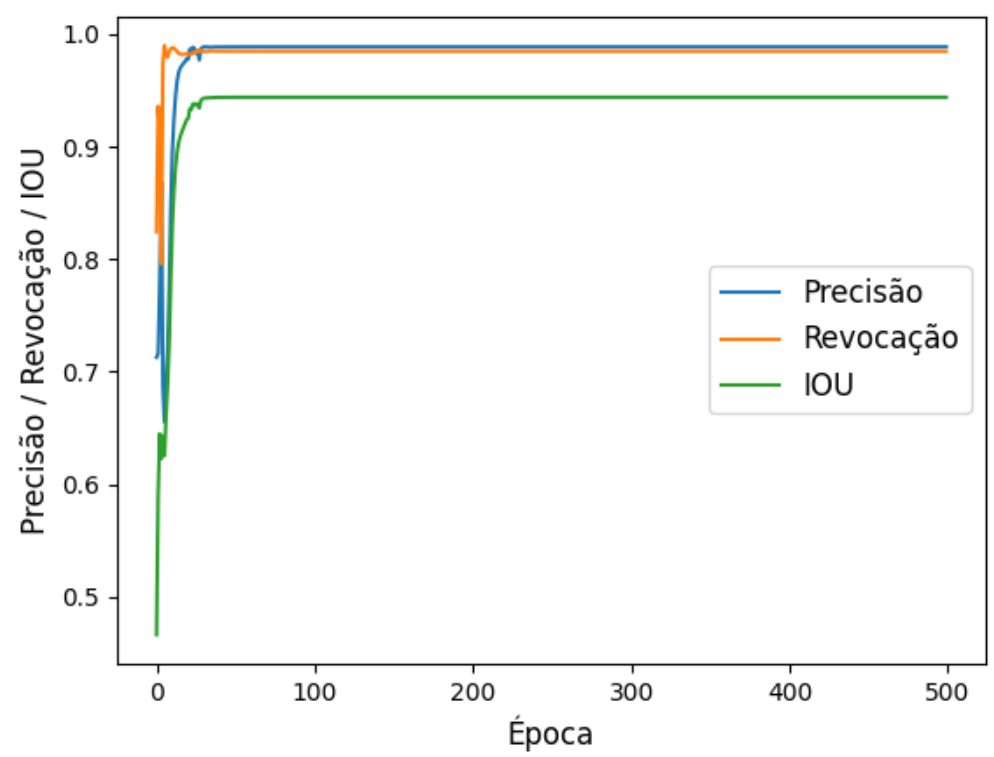

Fonte: autor.

Os resultados visuais do processo de segmentação podem ser vistos na Figura 5. Nesta Figura são apresentadas algumas imagens originais da base de dados DMR-UFF, em escala de tons de cinza (Figura 5 (a)), bem como seu respectivo groundtruth (Figura 5 (b)) desenvolvido por um especialista e disponibilizado para fins de comparação, e a máscara de segmentação automática criada a partir do método proposto (Figura 5 (c)).

A Tabela 1 contém um comparativo entre os resultados obtidos neste trabalho e os demais trabalhos da literatura, citados na Seção II. Embora tenha sido desenvolvida para aplicações biomédicas, a U-Net demonstra uma performance superior comparado com outros métodos. O método proposto atinge o estado-da-arte nas métricas avaliadas mostrando não só a eficiência das Redes Neurais Convolucionais mas como uma tendência em aplicar métodos de aprendizado de máquina na solução de tarefas complexas do processamento e da análise de imagens.

Tabela 1. Comparação entre alguns métodos propostos na literatura.

\begin{tabular}{cccccc}
\hline Autor & Metodologia & Acurácia & IOU & Precisão & Revocação \\
\hline [Motta 2010] & Limiarização & $96 \%$ & - & - & $88 \%$ \\
[Marques 2012] & Limiarização & $97 \%$ & - & - & $97 \%$ \\
[Baffa et al. 2016] & Limiarização & $96 \%$ & - & - & $98 \%$ \\
[Marques et al. 2016] & Limiarização & $96 \%$ & - & - & $97 \%$ \\
[Melo et al. 2017] & VGG-CNN & $95 \%$ & - & - & $97 \%$ \\
Método Proposto & U-Net CNN & $\mathbf{9 8 , 2 4 \%}$ & $\mathbf{9 4 , 3 8 \%}$ & $\mathbf{9 8 , 8 6 \%}$ & $\mathbf{9 8 , 4 6 \%}$ \\
\hline
\end{tabular}

Fonte: autor. 
Figura 5. Resultados obtidos na segmentação pelo método proposto
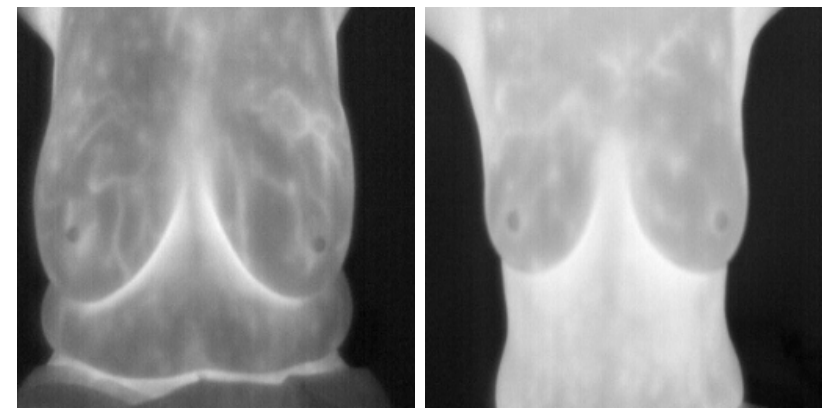

(a)
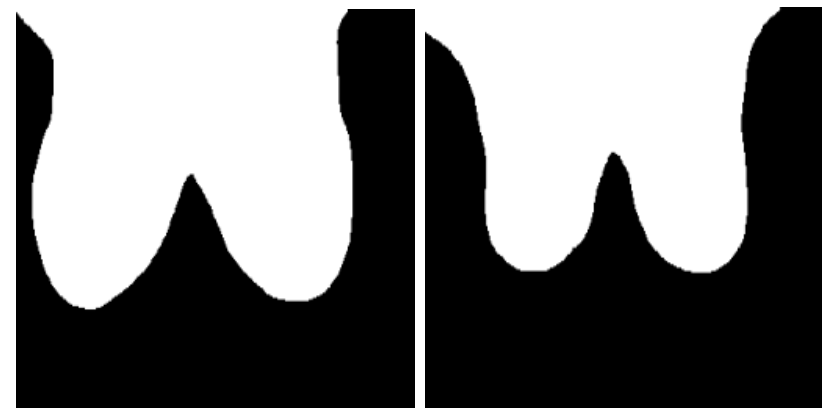

(b)
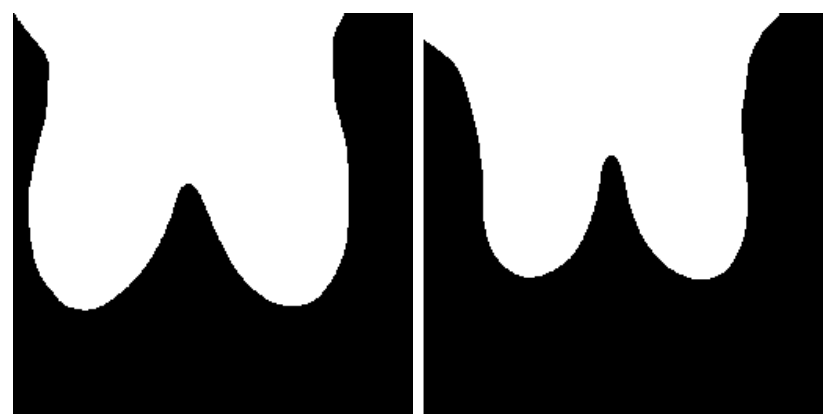

(c)
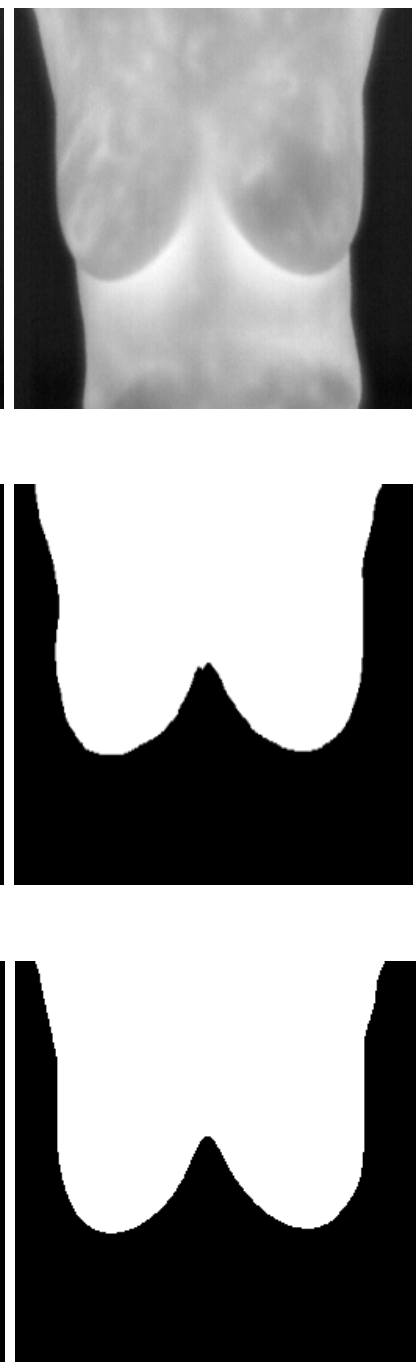

Fonte: autor.

Em (a) as imagens originais, em (b) as respectivas segmentações manuais realizadas por um especialista e em (c) as segmentações automáticas realizadas pelo algoritmo proposto.

\section{Conclusão}

O problema de segmentação de imagens infravermelhas da mama é comumente abordado na literatura como um problema de processamento de imagens. Diferente dos métodos tradicionalmente utilizados, este trabalho visa aplicar técnicas de aprendizado de máquina para que, a partir da detecção de regiões de interesse padrões em termografias mamárias, possa criar uma máscara de segmentação. Os resultados obtidos com a proposta atingem o estado da arte e, de forma satisfatória, contribui para o desenvolvimento de sistemas CADx eficientes. Espera-se que para trabalhos futuros, novas camadas com enfoque em realizar a classificação da imagem seja adicionado ao método para que, de forma única, o método possa segmentar, extrair padrões das mamas e classificar se um determinado exame é proveniente de um paciente doente ou saudável. 


\section{Agradecimentos}

A.C. é apoiada parcialmente pelo INCT (projeto MACC), pelo CNPq (projetos 402988 / 2016-7 e 305416 / 2018-9) e pela FAPERJ (projetos SIADE-2, e-Health Rio e Digit3D). M.B. é apoiado pela Coordenação de Aperfeiçoamento de Pessoal de Nível Superior Brasil (CAPES) (Projeto 88887.498626/2020-00). A.M.C. agradece ao FNDE pelo apoio na pesquisa junto ao Programa de Educação Tutorial (PET - Conexão de Saberes Ciência da Computação).

\section{Referências}

Amalu, W., Hobbins, W., Head, J., and Elliot, R. (2006). Infrared imaging of the breast-an overview. The Biomedical Engineering Handbook, 3rd ed., Medical Devices and Systems. CRC Press, Baton Rouge.

Baffa, M., Cheloni, D., and Lattari, L. (2016). Segmentaçao automática de imagens térmicas das mamas utilizando limiarizaçao com refinamento adaptativo. In Anais do XVI Workshop de Informática Médica, pages 39-48. SBC.

Baffa, M. F. O. and Lattari, L. G. (2018). Convolutional neural networks for static and dynamic breast infrared imaging classification. In 201831 st SIBGRAPI Conference on Graphics, Patterns and Images (SIBGRAPI), pages 174-181. IEEE.

Conci, A., Azevedo, E., and Leta, F. (2008). Computação Gráfica: Teoria e Prática, volume 2. Elsevier Editora.

Gore, J. P. and Xu, L. X. (2003). Thermal imaging for biological and medical diagnostics. Biomedical Photonics Handbook, 17:1-12.

IFF/Fiocruz. (2021). Câncer de mama: a importância do diagnóstico precoce. Disponível em: <http://www.iff.fiocruz.br/index.php/8-noticias/274-cancerdemama $>$. Acesso em: 05 abr. 2021.

Instituto Nacional do Cancer. (2021). Câncer de mama. Disponível em: $<$ https://www.inca.gov.br/tipos-de-cancer/cancer-de-mama $>$. Acesso em: 05 abr. 2021.

Ismael, A. M. and Şengür, A. (2021). Deep learning approaches for covid-19 detection based on chest X-ray images. Expert Systems with Applications, 164:114054.

Marques, R. S. (2012). Segmentação automática das mamas em imagens térmicas. Master's thesis, Instituto de Computação, Universidade Federal Fluminense, Niterói, RJ, Brasil.

Marques, R. S., Conci, A., Perez, M. G., Andaluz, V. H., and Mejia, T. M. (2016). An approach for automatic segmentation of thermal imaging in computer aided diagnosis. IEEE Latin America Transactions, 14(4):1856-1865.

Melo, R. H. C., Conci, A., and Vasconcelos, C. N. (2017). On the use of fully convolutional networks on evaluation of infrared breast image segmentations. In Anais do XVII Workshop de Informática Médica. SBC.

Motta, L. S. (2010). Obtenção automática da região de interesse em termogramas frontais da mama para o auxílio à detecção precoce de doenças. Master's thesis, Instituto de Computação, Universidade Federal Fluminense, Niterói, RJ, Brasil. 
Rehman, A., Khan, M. A., Saba, T., Mehmood, Z., Tariq, U., and Ayesha, N. (2021). Microscopic brain tumor detection and classification using $3 \mathrm{~d} \mathrm{cnn}$ and feature selection architecture. Microscopy Research and Technique, 84(1):133-149.

Ronneberger, O., Fischer, P., and Brox, T. (2015). U-net: Convolutional networks for biomedical image segmentation. In International Conference on Medical image computing and computer-assisted intervention, pages 234-241. Springer.

Silva, L., Saade, D., Sequeiros, G., Silva, A., Paiva, A., Bravo, R., and Conci, A. (2014). A new database for breast research with infrared image. Journal of Medical Imaging and Health Informatics, 4(1):92-100.

U.S. National Cancer Institute. (2021). Breast cancer screening (pdq) - patient version. Disponível em: $<$ https://www.cancer.gov/types/breast/patient/breast-screening-pdq $>$. Acesso em: 05 abr. 2021.

Zuluaga-Gomez, J., Zerhouni, N., Al Masry, Z., Devalland, C., and Varnier, C. (2019). A survey of breast cancer screening techniques: thermography and electrical impedance tomography. Journal of medical engineering \& technology, 43(5):305-322. 